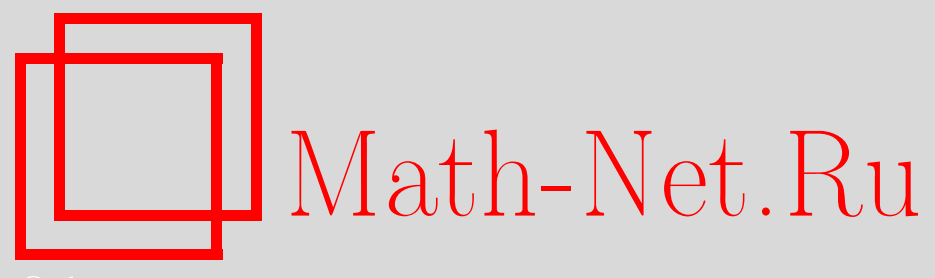

А. Е. Кирнасов, О склейке состояний автомата, Дискрет. матем., 2003, том 15, выпуск 4, 66-83

DOI: https://doi.org/10.4213/dm216

Использование Общероссийского математического портала Math-Net.Ru подразумевает, что вы прочитали и согласны с пользовательским соглашением http://www.mathnet.ru/rus/agreement

Параметры загрузки:

IP : 54.205 .225 .156

26 апреля 2023 г., 18:25:52 
УдК 519.7

\title{
О склейке состояний автомата
}

\author{
() 2003 г. А. Е. Кирнасов
}

В статье рассматривается задача о склейке состояний автомата, которая часто возникает при исследовании установочного эксперимента. Приводятся различные оценки времени склейки $r$ состояний автомата с $n$ состояниями.

Работа выполнена при поддержке Российского фонда фундаментальных исследований, проект 02-01-00162.

\section{1. Введение}

Одной из задач теории экспериментов с автоматами является установочная задача [1]. Это задача определения заключительного состояния автомата в процессе эксперимента, то есть опрашивания автомата некоторым множеством слов входного алфавита при условии, что до эксперимента полностью известна диаграмма автомата, но не известно начальное состояние. Если в процессе эксперимента выясняется, что текущим состоянием автомата является одно из состояний некоторого множества $S$, то на следующем шаге целесообразно либо опросить автомат словом, по реакции на которое множество $S$ можно будет нетривиальным образом разбить на подмножества, либо подать на вход автомата слово, переводящее два или более состояний множества $S$ в одно состояние. В [4] при построении простого безусловного установочного эксперимента вводится монотонно убывающая характеристика состояния эксперимента, в некотором смысле одновременно учитывающая обе описанные возможности. Первая возможность, связанная с разбиением множества $S$, сводится к понятию различимости состояний, которое хорошо изучено. Вторая возможность, связанная с переводом состояний в одно, приводит к необходимости изучения понятия склейки состояний. В настоящей статъе приводятся оценки максимального времени склейки $r$ состояний автомата с $n$ состояниями, а также асимптотика среднего времени склейки $r$ состояний автомата с $n$ состояниями при $r \leqslant \log _{2} \log _{2} \log _{2} n$.

\section{2. Основные понятия и результаты}

Пусть $\mathfrak{A}=(A, Q, B, \varphi, \psi)$ - автомат, где $A-$ входной алфавит, $B$ - выходной алфавит, $Q$ - множество состояний, $\varphi$ - функция переходов, $\psi$ - функция выходов. Мы также будем рассматривать естественное продолжение функции $\varphi$ на слова из алфавита $A$, которое также будем обозначать $\varphi$. Пусть теперь $q_{1}, q_{2}, \ldots, q_{r}$ - некоторые $r$ состояний автомата. Если существует слово $\alpha$ в алфавите $A$ такое, что $\varphi\left(q_{1}, \alpha\right)=\varphi\left(q_{2}, \alpha\right)=\ldots=\varphi\left(q_{r}, \alpha\right)$, то длину кратчайшего такого слова назовем временем склейки данных $r$ состояний. Введем в рассмотрение функцию $T_{\mathfrak{Y}}\left(q_{1}, q_{2}, \ldots, q_{r}\right)$, которая равна 0 , если состояния $q_{1}, q_{2}, \ldots, q_{r}$ 
автомата $\mathfrak{A}$ склеить нельзя, и равна времени склейки этих состояний в противном случае. Заметим, что для анализа склейки состояний, нам по существу нужна лишь диаграмма переходов автомата, поэтому в дальнейшем рассматриваются только автоматы без выхода. Пару $\left(\mathfrak{A},\left(q_{1}, q_{2}, \ldots, q_{r}\right)\right)$, состоящую из автомата $\mathfrak{A}$ и неупорядоченного набора его состояний назовем $r$-инициальным автоматом. Пусть $\mathfrak{B}=\left(\mathfrak{A},\left(q_{1}, q_{2}, \ldots, q_{r}\right)\right)-$ $r$-инициальный автомат. Тогда положим

$$
T(\mathfrak{B})=T_{\mathfrak{Y}}\left(q_{1}, q_{2}, \ldots, q_{r}\right) .
$$

Рассмотрим класс $\mathfrak{A}_{m, n}$ автоматов с $m$ входными символами и $n$ состояниями. Также рассмотрим класс

$\mathbb{\Re}_{m, n}^{\prime}=\left\{\mathfrak{U}=(A, Q, B, \varphi, \psi)|| A|=m| Q \mid,=n, \forall q_{1}, q_{2} \in Q \exists \alpha \in A^{*}: \varphi\left(q_{1}, \alpha\right)=\varphi\left(q_{2}, \alpha\right)\right\}$

Введем в рассмотрение функцию

$$
l(\mathcal{U}, r)=\max _{q_{1} \ldots, q_{r}} T_{\mathfrak{Y}}\left(q_{1}, \ldots, q_{r}\right)
$$

Теперь определим две функции

$$
\begin{aligned}
L(m, n, r) & =\max _{\mathfrak{Q} \in \mathbb{N}_{m, n}} l(\mathfrak{A}, r), \\
L^{\prime}(m, n, r) & =\max _{\mathfrak{H} \in \mathbb{N}_{m, n}^{\prime}} l(\mathfrak{A}, r) .
\end{aligned}
$$

Теорема 1. Справедливы неравенства

$$
\begin{aligned}
& L(m, n, r) \leqslant \sum_{i=1}^{r}\left(\begin{array}{l}
n \\
i
\end{array}\right) \\
& L(m, n, r)>\left(\frac{n-2 r}{r-1}\right)^{r-1}
\end{aligned}
$$

npu $m \geqslant r, n \geqslant 2 r$.

Теорема 2. Справедливы соотношения

$$
\begin{aligned}
L(m, n, 2) & =L^{\prime}(m, n, 2)=\frac{n(n-1)}{2}, \\
\frac{n^{2}(r-1)}{r}-2 n & \leqslant L^{\prime}(m, n, r) \leqslant \frac{(r-1) n(n-1)}{2}-\sum_{k=1}^{r}\left(\frac{k(k-1)}{2}-1\right) .
\end{aligned}
$$

Теорема 3. Пусть $n \rightarrow \infty$. Тогда для почти всех 2-иничиальных автономных автоматов (с одним входным символом) $\mathfrak{B}$ с п состояниями

$$
T(\mathfrak{B}) \leqslant \sqrt{n} \log _{2} n .
$$

Теорема 4. Пусть $n \rightarrow \infty, m \geqslant 2$ фиксировано и $r \leqslant \log _{2} \log _{2} \log _{2} n$. Тогда для почти всех $r$-инициальных автоматов $\mathfrak{B}$ с $m$ входными символами и п состояниями

$$
(r-1) \log _{m} n-4 \log _{2} \log _{2} n \leqslant T(\mathfrak{B}) \leqslant(r-1) \log _{m} n+\log _{2} \log _{2} \log _{2} \log _{2} \log _{2} n .
$$




\section{3. Доказательство теорем 1 и 2}

Докажем теорему 1 . Рассмотрим автомат $\mathfrak{A}=(A, Q, B, \varphi, \psi)$. Продолжим функщию $\varphi$ на подмножества множества состояний автомата, а именно, для $S \subseteq Q$ положим

$$
\varphi(S, \alpha)=\left\{q \in Q \mid \exists q^{\prime} \in Q: q=\varphi\left(q^{\prime}, \alpha\right)\right\}
$$

Неравенство

$$
L(m, n, r) \leqslant \sum_{i=2}^{r}\left(\begin{array}{l}
n \\
i
\end{array}\right)
$$

вытекает из того, что если $\alpha$ - кратчайшее слово такое, что

$$
\varphi\left(q_{1}, \alpha\right)=\varphi\left(q_{2}, \alpha\right)=\ldots=\varphi\left(q_{r}, \alpha\right)
$$

то для любых двух начальных подслов $\alpha_{1}$ и $\alpha_{2}$ слова $\alpha$ множества $\varphi\left(Q_{0}, \alpha_{1}\right)$ и $\varphi\left(Q_{0}, \alpha_{2}\right)$ различны и содержат не более $r$ элементов. Здесь $Q_{0}=\left\{q_{1}, \ldots q_{r}\right\}$.

Для доказательства нижней оценки мы несколько модифицируем одну из вариаџий конструкщия из [2], а именно, положим

$$
\begin{aligned}
p & =[(n-2) /(r-1)] \\
s & =n-2-p(r-1), \\
Q & =\left\{q_{0}, q_{0}^{\prime}\right\} \cup \bigcup_{i=1}^{r-1} Q_{i} \cup\left\{q_{1}^{\prime}, q_{2}^{\prime} \ldots, q_{s}^{\prime}\right\}
\end{aligned}
$$

где

$$
Q_{i}=\left\{q_{i, 1}, \ldots, q_{i, p}\right\}
$$

Очевидно, что $p \geqslant 2$. Положим также $A=\left\{a_{1}, \ldots, a_{m}\right\}$.

Рассмотрим теперь автомат $\mathfrak{A}=(A, Q, B, \varphi, \psi)$, где функцию $\varphi$ мы определим следующим образом:

- для всех $a \in A$

$$
\varphi\left(q_{0}, a\right)=q_{0}, \quad \varphi\left(q_{0}^{\prime}, a\right)=q_{0}^{\prime}, \quad \varphi\left(q_{i}^{\prime}, a\right)=q_{i}^{\prime}, \quad 1 \leqslant i \leqslant s
$$

- если $i>r$, то $\varphi\left(q, a_{i}\right)=q$ для всех $q$;

- если $j \leqslant p$, то $\varphi\left(q_{i, j}, a_{i}\right)=q_{i, j+1}$, и $\varphi\left(q_{i, p}, a_{i}\right)=q_{i, p}$;

- пусть $i_{0}<i$, тогда $\varphi\left(q_{i, j}, a_{i_{0}}\right)=q_{i, j}$ при любом $j$;

- пусть $i<i_{0} \leqslant m$, тогда для всех $j<p$

$$
\varphi\left(q_{i, j}, a_{i_{0}}\right)=q_{0}^{\prime}, \quad \varphi\left(q_{i, p}, a_{i_{0}}\right)=q_{i, 1}
$$

- наконец, $\varphi\left(q_{i, j}, a_{r}\right)=q_{0}^{\prime}$, если $j \neq p$ и $\varphi\left(q_{i, p}, a_{r}\right)=q_{0}$. 
Рассмотрим теперь множество $Q_{0}$, состоящее из $r$ состояний:

$$
Q_{0}=\left\{q_{0}, q_{1,1}, q_{2,1}, \ldots q_{r-1,1}\right\}
$$

Покажем, что любое слово, склеивающее состояния из $Q_{0}$, должно иметь длину не меньшую, чем $(p-1)^{r-1}+1$.

Пусть $\alpha$ - кратчайшее слово, склеивающее состояния из $Q_{0}$. Очевидно, его последним символом является $a_{r}$. Кроме того, если обозначить $\alpha^{\prime}$ начальный отрезок слова $\alpha$ без последнего символа, то нетрудно понять, что в этом слове нет символа $a_{r}$, а также что это слово является кратчайшим словом, переводящим множество $Q_{0}$ в множество $Q_{1}=\left\{q_{0}, q_{1, p}, q_{2, p}, \ldots q_{r-1, p}\right\}$. Индукцией по $m$ нетрудно проверить, что длина такого слова равна $(p-1)^{r-1}$. Значит, длина слова $\alpha$ равна $(p-1)^{r-1}+1$, что и требовалось доказать. Учитывая, что $p>(n-2) /(r-1)-1$, получаем нижнюю оценку теоремы 1 .

Прежде чем перейти к доказательству теоремы 2 , докажем следующую лемму.

Лемма 1. Пусть $\mathfrak{U}=(A, Q, B, \varphi, \psi)$ - автомат из класса $\mathfrak{i}_{m, n}^{\prime} u S \subseteq Q,|S|=k$. Тогда найдутся состояния $q_{1}$ и $q_{2}$ из $S$ такие, что существует слово $\alpha$ длины не большей, чем

$$
\frac{n(n-1)}{2}-\frac{k(k-1)}{2}+1 \text {, }
$$

склеиваючее эти состояния.

Доказательство. Пусть утверждение неверно, тогда при $q_{1}$ и $q_{2}$, произвольно выбранных из $S$, для длины $l$ кратчайшего слова $\alpha$, склеивающего $q_{1}$ и $q_{2}$, справедлива оценка

$$
l>l(n, k)=\frac{n(n-1)}{2}-\frac{k(k-1)}{2}+1 .
$$

Следовательно, начальными отрезками слова $\alpha$ пара состояний $\left\{q_{1}, q_{2}\right\}$ переводится в более, чем $l(n, k)$ различных пар, среди которых должно быть не менее, чем $l-l(n, k)>0$ пар, отличных от $\left\{q_{1}, q_{2}\right\}$ таких, что обе их компоненты лежат в $S$. Рассмотрим ту из этих пар, в которую пара $\left\{q_{1}, q_{2}\right\}$ переводится максимальным начальным отрезком слова $\alpha$. Длина такого отрезка должна быть не менее, чем $l-l(n, k)$, а длина слова, получающегося из слова $\alpha$ отбрасыванием этого начального отрезка, соответственно, не более, чем $l(n, k)$. Легко видеть, что это слово склеивает указанную пару состояний, обе компоненты которой лежат в $S$. Получаемое противоречие со сделанным предположением завершает доказательство леммы.

Перейдем к доказательству теоремы 2. Легко видеть, что верхняя оценка теоремы получается из леммы 1 индукщией по $r$.

Рассмотрим автомат $\mathfrak{U}=(A, Q, B, \varphi, \psi)$ с двумя входными символами, где

$$
\begin{aligned}
& A=\{0,1\}, \quad Q=\{1,2, \ldots, 3\}, \\
& \varphi(i, 1)=i \quad \text { при } i \neq 1, \quad \varphi(1,1)=2 \text {, } \\
& \varphi(i, 0)=i+1 \text { при } i \neq n, \varphi(n, 0)=1 \text {. }
\end{aligned}
$$

Заметим, что аналогичный пример автомата был построен П. А. Пантелеевым при рассмотрении различных обобщений понятия различимости состояний.

Пусть $S \subseteq Q, S=\left\{i_{1}, i_{2}, \ldots i_{k}\right\}$. Можно считать, что $i_{1}<i_{2}<\ldots<i_{k-1}<i_{k}$. Введем числа $j_{s}=i_{s+1}-i_{s}$ при $s<k, j_{k}=n-i_{k}+i_{1}$ и положим

$$
d=\max _{1 \leqslant s \leqslant k} \max j_{s}
$$


Пусть $\alpha$ - слово, склеивающее все состояния из $S$ и $l$ - длина этого слова. Докажем, что $l \geqslant 1+n(n-d-1)$.

Рассмотрим последний символ 1 в слове $\alpha$, а также слово $\alpha^{\prime}$, получающееся из $\alpha$ отбрасыванием заключительного отрезка, начинающегося с этого символа. Индукцией по $k$ можно проверить, что найдется такое $t, 1 \leqslant t \leqslant k$, что $\varphi\left(i_{t}, \alpha^{\prime}\right)=1, \varphi\left(i_{t-1}, \alpha^{\prime}\right)=2$ при $t \neq 1, \varphi\left(i_{k}, \alpha^{\prime}\right)=2$ при $t=1$. Выделим минимальный начальный отрезок $\beta$ слова $\alpha^{\prime}$, переводящий $i_{t}$ в 2 , заканчивающийся символом 1 . Такой отрезок, очевидно, найдется. При этом нетрудно понять, что при $t \neq 1$

$$
\varphi\left(i_{t-1}, \beta\right) \geqslant 2+\left(n-j_{t}-1\right)
$$

и при $t=1$

$$
\varphi\left(i_{k}, \beta\right) \geqslant 2+\left(n-j_{t}-1\right) .
$$

Если обозначить $\gamma$ заключительный отрезок слова $\alpha^{\prime}$, получающийся из него отбрасыванием слова $\beta$, то получим, что для некоторого

$$
r \geqslant 2+\left(n-j_{t}-1\right) \geqslant 2+(n-d-1)
$$

справедливы равенства

$$
\varphi(2, \beta)=1, \quad \varphi(r, \beta)=2 .
$$

Индукцией по $r$ можно установить, что длина слова $\beta$ не меньше, чем $n(r-2)$. Отсюда нетрудно заключить, что $l \geqslant 1+n(n-d-1)$.

Положим теперь

$$
\begin{gathered}
d=[n / r], \quad q=n-r d, \\
i_{1}=1, i_{2}=i_{1}+d+1, \ldots i_{q+1}=i_{q}+d+1, i_{q+2}=i_{q+1}+d, \ldots i_{r}=i_{r-1}+d .
\end{gathered}
$$

Применяя только что доказанное утверждение к множеству состояний $\left\{i_{1}, \ldots i_{r}\right\}$, получаем для длины $l$ кратчайшего склеивающего слова оценку $l \geqslant 1+n(n-d-2)$. Так как $d \leqslant n / r$, получаем, наконец, что

$$
L^{\prime}(m, n, r) \geqslant \frac{n^{2}(r-1)}{r}-2 n .
$$

Несколько уточняя предыдущие рассмотрения, можно проверить, что длина кратчайшего склеивающего слова для пары состояний $\{2,2+[n / 2]\}$ в описанном автомате равна $n(n-1) / 2$. Это заканчивает проверку равенства

$$
L(m, n, 2)=L^{\prime}(m, n, 2)=\frac{n(n-1)}{2}
$$

и доказательство теоремы 2.

\section{4. Доказательство теоремы 3}

В этом разделе мы рассмотрим класс $K_{n}$ автоматов с одним входным символом (автономные автоматы) и $n$ состояниями. Поставим следующий вопрос: при каком $T$, зависящем от $n$, вероятность того, что в случайно выбранном автомате из $K_{n}$ время склейки двух случайно выбранных состояний этого автомата не меньше $T$, стремится к нулю, когда $n$ стремится к бесконечности? Покажем, что достаточно положить

$$
T=\sqrt{n} \log _{2} n .
$$


Обозначим $D(n)$ множество диаграмм переходов, задающих автономные автоматы. Обозначим также $D(n, T)$ множество диаграмм переходов, задающих автономные автоматы, для которых два фиксированных состояния склеиваются за время, не меньшее, чем $T$. Нетрудно понять, что приведенное утверждение вытекает теперь из следующей теоремы.

Теорема 5. При $n \rightarrow \infty$

$$
\frac{\left|D\left(n, \sqrt{n} \log _{2} n\right)\right|}{|D(n)|} \rightarrow 0 .
$$

Доказательство. Заметим, что для автоматов из $K_{n}$ время склейки двух состояний не превосходит $n$, если эти состояния могут быть склеены. Зафиксируем два состояния $q_{1}$ и $q_{2}$ и подсчитаем число диаграмм, задающих автоматы из $K_{n}$, в которых кратчайшее слово, склеивающее $q_{1}$ и $q_{2}$, имеет длину $t$ при $1 \leqslant t \leqslant n$. Класс всех таких диаграмм обозначим $D\left(n, t, q_{1}, q_{2}\right)$. Введем обозначение

$$
\left|D\left(n, t, q_{1}, q_{2}\right)\right|=S(n, t) .
$$

Рассмотрим некоторую диаграмму из $D\left(n, t, q_{1}, q_{2}\right)$. Обозначим $f$ функщию переходов, соответствующую этой диаграмме, то есть $f(q)$ для произвольного $q$ обозначает состояние, в которое идет единственная стрелка из $q$. Рассмотрим последовательность

$$
\alpha_{1}=\left\{q_{1}^{1}=q_{1}, q_{1}^{2}=f\left(q_{1}^{1}\right), \ldots, q_{1}^{r}=f\left(q_{1}^{r-1}\right)\right\}
$$

такую, что $f\left(q_{1}^{r}\right)=q_{1}^{s}$, где $s \leqslant r$ и $q_{1}^{i} \neq q_{1}^{j}$ при $r \geqslant i>j$. Такая последовательность единственна. Множество состояний из этой последовательности обозначим $Q_{1}$. Возможны два варианта, $q_{2} \in Q_{1}$ и $q_{2} \notin Q_{1}$. Множество диаграмм, на которых реализуется первый вариант, обозначим $D_{1}\left(n, t, q_{1}, q_{2}\right)$. Множество диаграмм из $D\left(n, t, q_{1}, q_{2}\right)$, на которых реализуется второй вариант, обозначим $D_{2}\left(n, t, q_{1}, q_{2}\right)$. Рассмотрим диаграмму из $D_{1}\left(n, t, q_{1}, q_{2}\right)$. Определим последовательность

$$
\alpha_{2}=\left\{q_{2}^{1}=q_{2}, q_{2}^{2}=f\left(q_{2}\right), \ldots, q_{z}^{2}=f\left(q_{z-1}^{2}\right)\right\}
$$

такую, что $q_{2}^{i} \neq q_{2}^{J}$ при $z \geqslant i>j, f\left(q_{2}^{z}\right)=q_{1}^{p}$ для некоторого $p \leqslant r, q_{2}^{i} \notin Q_{1}$ при $i \leqslant z$. Такая последовательность также единственна и множество составляющих ее состояний обозначим $Q_{2}$. Возможны три случая, $z=t ; z \neq t, p-1>z$ и $z \neq t, p-1<z$.

Обозначим множества диаграмм из $D_{1}\left(n, t, q_{1}, q_{2}\right)$, на которых реализуются эти три случая соответственно $D_{1}^{1}\left(n, t, q_{1}, q_{2}\right), D_{1}^{2}\left(n, t, q_{1}, q_{2}\right)$ и $D_{1}^{3}\left(n, t, q_{1}, q_{2}\right)$. Введем обозначение

$$
\begin{array}{ll}
\left|D_{1}^{1}\left(n, t, q_{1}, q_{2}\right)\right|=S_{1}(n, t), & \left|D_{1}^{2}\left(n, t, q_{1}, q_{2}\right)\right|=S_{2}(n, t), \\
\left|D_{1}^{3}\left(n, t, q_{1}, q_{2}\right)\right|=S_{3}(n, t), & \left|D_{2}\left(n, t, q_{1}, q_{2}\right)\right|=S_{4}(n, t) .
\end{array}
$$

Очевидно,

$$
S(n, t)=S_{1}(n, t)+S_{2}(n, t)+S_{3}(n, t)+S_{4}(n, t) .
$$

Найдем $S_{1}(n, t)$. Диаграмма из $D_{1}^{1}\left(n, t, q_{1}, q_{2}\right)$ однозначно определяется выбором $r$, упорядоченной выборкой $r-1$ состояний из $n-2$, которые вместе с $q_{1}$ составят последовательность $\alpha_{1}$, выбором $s$ в пределах от 1 до $r$ и упорядоченной выборкой $t-1$ состояний из $n-r-1$, которые вместе с $q_{1}$ составят последовательность $\alpha_{2}$, и фиксацией отображения $f$ на оставшихся $n-r-t$ состояниях. Таким образом,

$$
S_{1}(n, t)=\sum_{r=1}^{n-1} \sum_{s=1}^{r} A_{n-2}^{r+t-2} n^{n-r-t},
$$


где

$$
A_{m}^{k}=m(m-1) \ldots(m-k+1)
$$

Подсчитаем $S_{2}(n, t)$. Нетрудно понять, что для диаграмм из класса $D_{2}^{1}\left(n, t, q_{1}, q_{2}\right)$ выполняются следующие условия: $p-z-1$ делит $r-s+1 ; t=s-1$.

Фиксацию диаграммы из $D_{2}^{1}\left(n, t, q_{1}, q_{2}\right)$ можно таким образом осуществить так: выбираем $r$; затем берем упорядоченную выборку $r-1$ состояний из $n-2$, которые составят вместе с $q_{1}$ последовательность $\alpha_{1}$; затем фиксируем $s=t-1$, если $t-1 \leqslant r$; затем выбираем $z \leqslant t-1$ и $p$ от 2 до $r$ так, что $r-s+1$ делит $p-z-1$; наконец, берем упорядоченную выборку $z-1$ упорядоченных состояний из $n-r-1$, которые вместе с $q_{2}$ составят $\alpha_{2}$ и произвольно определяем $f$ на оставшихся $n-r-z$ состояниях. Таким образом,

$$
S_{2}(n, t)=\sum_{r=t-1}^{n-1} \sum_{z=1}^{t-1} \sum_{p=2}^{r} A_{n-2}^{z-1} n^{n-r-z} \delta(r-s+1, p-z-1),
$$

где $\delta(n, m)=1$, если $n$ делит $m$ и $\delta(n, m)=0$ в остальных случаях.

Рассуждая аналогичным образом, получаем, что

$$
\begin{aligned}
& S_{3}(n, t)=\sum_{r=1}^{n-1} \sum_{s=1}^{r} \sum_{z=1}^{t-1} A_{n-2}^{z-1} n^{n-r-z} \delta(r-s+1, t-s+1), \\
& S_{4}(n, t)=\sum_{r=t+1}^{n} \sum_{p=1}^{r-1} A_{n-2}^{r-2} * n^{n-r} \delta(r-s+1, t-r+p) .
\end{aligned}
$$

Путем алгебраических преобразований получаем следующее более простое выражение для $S(n, t)$ :

$$
S(n, t)=2\left(S_{5}(n, t)+S_{6}(n, t)\right)+S_{7}(n, t),
$$

где

$$
\begin{aligned}
& S_{5}(n, t)=\sum_{p=1}^{n-t-1} \sum_{z=1}^{t-1} \sum_{k=1}^{[(t+p-z-1) / p]} A_{n-2}^{t+p+z-2} n^{n-t-p-z} \\
& S_{6}(n, t)=\sum_{p=1}^{n-t} \sum_{k=1}^{[(t+p-1) / p]} A_{n-2}^{t+p-2} n^{n-t-p}, \\
& S_{7}(n, t)=\sum_{r=1}^{n-1} \sum_{s=t+1}^{r} A_{n-2}^{r+t-2} n^{n-r-t}
\end{aligned}
$$

Перейдем теперь к оценкам. Во-первых, отметим, что

$$
S(n, t) \leqslant 2 S_{8}(n, t)+S_{7}(n, t),
$$

где

$$
S_{8}(n, t)=\sum_{p=1}^{n-t} \sum_{z=0}^{t-1} \sum_{k=1}^{[(t+p-z-1) / p]} A_{n-2}^{t+p+z-2} n^{n-t-p-z}
$$


Учтем теперь, что число всех диаграмм переходов, соответствующих автоматам с $n$ состояниями и одним входом, равно $n^{n}$. Нетрудно получить, исходя из формул для $S_{7}(n, t)$ и $S_{8}(n, t)$, что

$$
\begin{aligned}
& \frac{S_{8}(n, t)}{n^{n}} \leqslant \prod_{i=2}^{t}\left(1-\frac{i}{n}\right), \\
& \frac{S_{7}(n, t)}{n^{n}} \leqslant \prod_{i=2}^{t}\left(1-\frac{i}{n}\right) .
\end{aligned}
$$

Отсюда

$$
\frac{S(n, t)}{n^{n}} \leqslant 3 \prod_{i=2}^{t}\left(1-\frac{i}{n}\right) .
$$

Оценим последнее произведение. Ясно, что

$$
\ln \prod_{i=2}^{t}\left(1-\frac{i}{n}\right)=\sum_{i=2}^{t} \ln \left(1-\frac{i}{n}\right) \leqslant-\sum_{i=2}^{t} \frac{i}{n} \leqslant-\frac{t^{2}}{3 n} .
$$

Отсюда

$$
\frac{1}{n^{n}} \sum_{t=T}^{n} S(n, t) \leqslant 3 n \exp \left(-\frac{T^{2}}{3 n}\right) .
$$

Положив $T=\sqrt{n} \log _{2} n$, получим, что при $n \rightarrow \infty$

$$
\frac{1}{n^{n}} \sum_{t=T}^{n} S(n, t) \rightarrow 0
$$

Это завершает доказательство теоремы 3.

\section{5. Вспомогательные определения и леммы}

В этом и следующем разделах мы рассматриваем автоматы с $m \geqslant 2$ входными символами. Зафиксируем $m \geqslant 2, n$, а также множества

$$
A=\left\{a_{1}, \ldots, a_{m}\right\}, \quad Q=\left\{q_{1}, \ldots q_{n}\right\}
$$

которые мы будем называть множествами входных символов и вершин соответственно. Обозначим $D(n, m)$ множество помеченных ориентированных графов с множеством вершин $Q$ таких, что из каждой вершины исходит $m$ ребер, помеченных элементами множества $A$ так, что разные ребра, исходящие из одной вершины, помечены разными элементами. Свяжем с каждой диаграммой $P$ из $D(n, m)$ функцию $f_{P}: Q \times A \rightarrow Q$, которую назовем функцией переходов графа $P$. Для каждого $q \in Q$ и для каждого $a \in A$ положим $f_{P}(q, a)=q^{\prime}$, где $q^{\prime}-$ та из вершин, куда ведет ребро с началом в $q$, помеченное символом $a$. Продолжим далее обычным образом функщию переходов $f$ на слова из алфавита $A$ и за этой новой функщией сохраним прежнее обозначение.

Зафиксируем граф $P$, вершину $q \in Q$ и слово $\alpha$ в алфавите $A$ длины $t$. Путем, исходящим из вершины $q$, определяемым словом $\alpha$, мы назовем последовательность, 
состоящую из чередующихся вершин и ребер, определяемую следующим образом: первой вершиной пути является вершина $q$, первым ребром пути, следующим за этой вершиной, является то из ребер, исходящих из вершины $q$, которое помечено первым символом слова $\alpha ;(i+1)$-й по порядку вершиной пути будет вершина $f\left(q, \alpha^{i}\right)$, где $\alpha^{i}$ есть начальный отрезок слова $\alpha$ длины $i$; наконец, $i$-м ребром пути, следующим за $i$-й вершиной, является то из ребер, исходящих из $i$-й вершины, которое помечено $i$-м символом слова $\alpha$, при этом $i$ меняется от 1 до $t$. Обозначим теперь путь, исходящий из вершины $q$, определяемый словом $\alpha$ через $\pi(q, \alpha)$. Выделим из пути $\pi(q, \alpha)$ подпоследовательность, состоящую только из вершин, которую назовем вершинным путем, начинающимся в вершине $q$ и определяемым словом $\alpha$. Этот путь обозначим $\pi^{\prime}(q, \alpha)$. Наконец, множество вершин, содержащихся в последовательности $\pi^{\prime}(q, \alpha)$, обозначим $\tilde{\pi}(q, \alpha)$.

Пусть $T>1$. Рассмотрим подкласс $D^{\prime}(n, m, T)$ класса $D(n, m, T)$, состоящий из таких графов $P$, что найдется слово $\alpha$ длины $[T]$, для которого вершинные пути $\pi^{\prime}\left(q_{1}, \alpha\right)$, $\pi^{\prime}\left(q_{2}, \alpha\right), \ldots, \pi^{\prime}\left(q_{r}, \alpha\right)$ состоят каждый из попарно разных вершин и пересекаются только по концевой вершине. Оценим снизу число графов в $D^{\prime}(n, m, T)$. Для каждого неупорядоченного набора $\left(\alpha_{i_{1}}, \alpha_{i_{2}}, \ldots, \alpha_{i_{k}}\right)$ из $k$ различных слов длины [T] выделим в классе $D^{\prime}(n, m, T)$ подкласс $D^{\prime}\left(n, m, T,\left(\alpha_{i_{1}}, \alpha_{i_{2}}, \ldots, \alpha_{i_{k}}\right)\right)$, состоящий из таких графов $P$, что для каждого $1 \leqslant j \leqslant k$ вершинные пути $\pi^{\prime}\left(q_{1}, \alpha_{i_{j}}\right), \pi^{\prime}\left(q_{2}, \alpha_{i_{j}}\right) \ldots, \pi^{\prime}\left(q_{r}, \alpha_{i_{j}}\right)$ состоят каждый из попарно разных вершин и пересекаются только по конщевой вершине.

Для каждого набора $\left(\alpha_{i_{1}}, \alpha_{i_{2}}, \ldots, \alpha_{i_{k}}\right)$ разобьем $D^{\prime}\left(n, m, T,\left(\alpha_{i_{1}}, \alpha_{i_{2}}, \ldots, \alpha_{i_{k}}\right)\right)$ на три подкласса $D_{1}^{\prime}\left(n, m, T,\left(\alpha_{i_{1}}, \alpha_{i_{2}}, \ldots, \alpha_{i_{k}}\right)\right), \quad D_{2}^{\prime}\left(n, m, T,\left(\alpha_{i_{1}}, \alpha_{i_{2}}, \ldots, \alpha_{i_{k}}\right)\right)$, $D_{3}^{\prime}\left(n, m . T,\left(\alpha_{i_{1}}, \alpha_{i_{2}}, \ldots, \alpha_{i_{k}}\right)\right)$. Для этого введем параметр $\operatorname{gl}\left(P,\left(\alpha_{i_{1}}, \alpha_{i_{2}}, \ldots, \alpha_{i_{k}}\right)\right)$ графа $P$ из $D^{\prime}\left(n, m, T,\left(\alpha_{i_{1}}, \alpha_{i_{2}}, \ldots, \alpha_{i_{k}}\right)\right)$ относительно множества слов $\left(\alpha_{i_{1}}, \alpha_{i_{2}}, \ldots, \alpha_{i_{k}}\right)$. Введем обозначение

$$
S=\bigcup_{j=1}^{k} \tilde{\pi}\left(q_{1}, \alpha_{i_{j}}\right) \cup \bigcup_{j=1}^{k} \tilde{\pi}\left(q_{2}, \alpha_{i_{j}}\right) \cup \ldots \cup \bigcup_{j=1}^{k} \tilde{\pi}\left(q_{r}, \alpha_{i_{j}}\right) .
$$

Для каждой вершины $q \in S$ обозначим через $n(q)$ уменьшенное на единицу число таких ребер, входящих в $q$, что каждое из этих ребер встречается хотя бы в одном из путей $\pi\left(q_{s}, \alpha_{i_{j}}\right), 1 \leqslant j \leqslant k, 1 \leqslant s \leqslant r$. Теперь положим

$$
\operatorname{gl}\left(P,\left(\alpha_{i_{1}}, \alpha_{i_{2}}, \ldots, \alpha_{i_{k}}\right)\right)=\sum_{q \in S} n(q)
$$

Содержательно этот параметр показывает общее число пересечений путей $\pi\left(q_{s}, \alpha_{i_{j}}\right)$, $1 \leqslant j \leqslant k, 1 \leqslant s \leqslant r$.

Определим теперь подкласс $D_{1}^{\prime}\left(n, m, T,\left(\alpha_{i_{1}}, \alpha_{i_{2}}, \ldots, \alpha_{i_{k}}\right)\right)$ следующим образом. Граф $P$ принадлежит подклассу $D_{1}^{\prime}\left(n, m, T,\left(\alpha_{i_{1}}, \alpha_{i_{2}}, \ldots, \alpha_{i_{k}}\right)\right)$ тогда и только тогда, когда $P \in D^{\prime}\left(n, m, T,\left(\alpha_{i_{1}}, \alpha_{i_{2}}, \ldots, \alpha_{i_{k}}\right)\right)$ и выполняется следующее условие: для любых $j_{1}$ и $j_{2}, 1 \leqslant j_{1}<j_{2} \leqslant k$, если $\alpha^{i_{j_{1} j_{2}}}$ обозначает максимальный общий начальный отрезок слов $\alpha_{i_{1}}$ и $\alpha_{i_{j_{2}}}$, то

$$
\begin{array}{r}
\left(\tilde{\pi}\left(q_{1}, \alpha_{i_{j_{1}}}\right) \cup \tilde{\pi}\left(q_{2}, \alpha_{i_{j_{1}}}\right) \cup \ldots \cup \tilde{\pi}\left(q_{r}, \alpha_{i_{j_{1}}}\right)\right) \cap\left(\tilde{\pi}\left(q_{1}, \alpha_{i_{j_{2}}}\right) \cup \tilde{\pi}\left(q_{2}, \alpha_{i_{j_{2}}}\right) \cup \ldots \cup \tilde{\pi}\left(q_{r}, \alpha_{i_{j_{2}}}\right)\right) \\
=\left(\tilde{\pi}\left(q_{1}, \alpha^{i_{j_{1} j_{2}}}\right) \cup \tilde{\pi}\left(q_{2}, \alpha^{i_{j_{1} j_{2}}}\right) \cup \ldots \tilde{\pi}\left(q_{r}, \alpha^{i_{j_{1}} j_{2}}\right)\right) .
\end{array}
$$

Определим теперь два оставшихся подкласса $D_{2}^{\prime}\left(n, m, T,\left(\alpha_{i_{1}}, \alpha_{i_{2}}, \ldots, \alpha_{i_{k}}\right)\right)$ и $D_{3}^{\prime}\left(n, m, T,\left(\alpha_{i_{1}}, \alpha_{i_{2}}, \ldots, \alpha_{i_{k}}\right)\right)$. 
Граф $P$, принадлежащий классу $D^{\prime}\left(n, m, T,\left(\alpha_{i_{1}}, \alpha_{i_{2}}, \ldots, \alpha_{i_{k}}\right)\right)$, принадлежит подклассу $D_{2}^{\prime}\left(n, m, T,\left(\alpha_{i_{1}}, \alpha_{i_{2}}, \ldots, \alpha_{i_{k}}\right)\right)$, если $\operatorname{gl}(P)>k(r-1)$. Заметим, что если $P \in D_{1}^{\prime}\left(n, m, T,\left(\alpha_{i_{1}}, \alpha_{i_{2}}, \ldots, \alpha_{i_{k}}\right)\right)$, то $\operatorname{gl}(P)=k(r-1)$, таким образом, подклассы $D_{1}^{\prime}\left(n, m, T,\left(\alpha_{i_{1}}, \alpha_{i_{2}}, \ldots, \alpha_{i_{k}}\right)\right)$ и $D_{2}^{\prime}\left(n, m, T,\left(\alpha_{i_{1}}, \alpha_{i_{2}}, \ldots, \alpha_{i_{k}}\right)\right)$ не пересекаются.

Наконец, в последний подкласс $D_{3}^{\prime}\left(n, m, T,\left(\alpha_{i_{1}}, \alpha_{i_{2}}, \ldots, \alpha_{i_{k}}\right)\right)$ мы отнесем графы из $D^{\prime}\left(n, m, T,\left(\alpha_{i_{1}}, \alpha_{i_{2}}, \ldots, \alpha_{i_{k}}\right)\right)$, не вошедшие в первые два подкласса.

Докажем следующую лемму.

Лемма 2. Пусть $P \in D_{3}^{\prime}\left(n, m, T,\left(\alpha_{i_{1}}, \alpha_{i_{2}}, \ldots, \alpha_{i_{k}}\right)\right)$. Тогда $P \in D_{2}^{\prime}(n, m, T, W)$ для некоторого строгого подмножества $W$ множества слов $\left(\alpha_{i_{1}}, \alpha_{i_{2}}, \ldots, \alpha_{i_{k}}\right)$.

Доказательство. Доказательство проведем индукцией по $k$. При $k=1$ это проверяется непосредственно. Пусть лемма доказана при всех $k<k_{0}$. Рассмотрим граф $P \in D_{3}^{\prime}\left(n, m, T,\left(\alpha_{i_{1}}, \alpha_{i_{2}}, \ldots, \alpha_{i_{k}}\right)\right)$. Допустим, что предположение леммы неверно, тогда для любого строгого подмножества $W$ множества слов $\left(\alpha_{i_{1}}, \alpha_{i_{2}}, \ldots, \alpha_{i_{k}}\right)$, либо $P \in D_{3}^{\prime}(n, m, T, W)$, либо $P \in D_{1}^{\prime}(n, m, T, W)$, но первый вариант невозможен, так как в противном случае согласно предположению индукции нашлось бы множество $W^{\prime}$, являющееся строгим подмножеством $W$, а значит, и исходного множества $\left(\alpha_{i_{1}}, \alpha_{i_{2}}, \ldots, \alpha_{i_{k}}\right)$, для которого $P \in D_{2}^{\prime}\left(n, m, T, W^{\prime}\right)$, что противоречит нашему допущению об отсутствии подобных подмножеств в множестве $\left(\alpha_{i_{1}}, \alpha_{i_{2}}, \ldots, \alpha_{i_{k}}\right)$. Следовательно, всегда имеет место второй случай, в частности, $P \in D_{1}^{\prime}\left(n, m, T,\left(\alpha_{i_{1}}, \alpha_{i_{2}}, \ldots, \alpha_{i_{k-1}}\right)\right)$. Отсюда нетрудно получить, что $P \in D_{1}^{\prime}\left(n, m, T,\left(\alpha_{i_{1}}, \alpha_{i_{2}}, \ldots, \alpha_{i_{k}}\right)\right)$, что противоречит допущению о том, что $P \in D_{3}^{\prime}\left(n, m, T,\left(\alpha_{i_{1}}, \alpha_{i_{2}}, \ldots, \alpha_{i_{k}}\right)\right)$.

Лемма доказана.

Верно также следующее более сильное утверждение.

Лемма 3. Пусть $P \in D_{3}^{\prime}\left(n, m, T,\left(\alpha_{i_{1}}, \alpha_{i_{2}}, \ldots, \alpha_{i_{k}}\right)\right)$. Тогда $P \in D_{2}^{\prime}(n, m, T, W)$ для некоторого строгого подмножества $W$ множества слов $\left(\alpha_{i_{1}}, \alpha_{i_{2}}, \ldots, \alpha_{i_{k}}\right)$, и если $\alpha_{i_{j}}$ не входит в $W$, то хотя бы один из путей $\pi\left(q_{1}, \alpha_{i_{j}}\right), \pi\left(q_{2}, \alpha_{i_{j}}\right), \ldots, \pi\left(q_{r}, \alpha_{i_{j}}\right)$ может быть получен комбиначией частей путей $\pi\left(q_{1}, \beta\right), \pi\left(q_{2}, \beta\right) \ldots, \pi\left(q_{r}, \beta\right)$ для $\beta \in W$.

Доказательство. Согласно лемме 1 найдется подмножество $W \in\left(\alpha_{i_{1}}, \alpha_{i_{2}}, \ldots, \alpha_{i_{k}}\right)$ такое, что $P \in D_{2}^{\prime}(n, m, T, W)$. Рассмотрим те слова из $\left(\alpha_{i_{1}}, \alpha_{i_{2}}, \ldots, \alpha_{i_{k}}\right)$, которые не вошли в $W$. Если $W$ не удовлетворяет условиям леммы 2 , то среди указанных слов найдется слово $\beta$, для которого пути $\pi\left(q_{1}, \beta\right), \pi\left(q_{2}, \beta\right), \ldots, \pi\left(q_{r}, \beta\right)$ не могут быть получены какой-либо комбинацией путей $\pi\left(q_{1}, \gamma\right)$ и $\pi\left(q_{2}, \gamma\right)$ для $\gamma \in W$. Положим тогда $W^{\prime}=W \cup\{\beta\}$. Нетрудно понять, что $P \in D_{2}^{\prime}\left(n, m, T, W^{\prime}\right)$. Если $W^{\prime}$ вновь не удовлетворяет условиям леммы 2 , то повторим рассуждения. Так как $P \in D_{3}^{\prime}\left(n, m, T,\left(\alpha_{i_{1}}, \alpha_{i_{2}}, \ldots, \alpha_{i_{k}}\right)\right)$, указанный процесс должен оборватъся построением множества с требуемыми свойствами.

Нам потребуется также следующая лемма.

Лемма 4. Пусть $P \in D_{3}^{\prime}(n, m, T, V)$ для некоторого множества из $k \leqslant d$ слов в алфавите $A$ u $\operatorname{gl}(P, V) \leqslant d$. Тогда существует не более, чем $2^{((r d+r) d)^{(r d+r) d}}$ множеств $W$ таких, что $V \subset W, P \in D^{\prime}(n, m, T, W)$, и хотя бы один из путей $\pi\left(q_{1}, \beta\right), \pi\left(q_{2}, \beta\right), \ldots, \pi\left(q_{r}, \beta\right)$ для слов $\beta \in W$ может быть получен комбиначией частей путей $\pi\left(q_{s}, \alpha\right)$ для $\alpha \in V$, $1 \leqslant s \leqslant r$.

Доказательство. Так как $\operatorname{gl}(P, V) \leqslant d$, каждый путь $\pi\left(q_{s}, \alpha\right), 1 \leqslant s \leqslant r$, разбивается точками пересечения с другими путями не более, чем на $d+1$ частей. Стало быть всего 
возникает не более, чем $(r d+r) d$ частей, из которых может быть составлен путь $\pi\left(q_{s}, \beta\right)$ при некотором $s, 1 \leqslant s \leqslant r$. Так как $P \in D^{\prime}(n, m, T, W)$, вершинный путь $\pi^{\prime}\left(q_{s}, \beta\right)$ не имеет повторяющихся вершин, и следовательно, комбинировать для получения $\pi\left(q_{s}, \beta\right)$ мы можем лишь различными частями путей из $(r d+r) d$ возможных. Отсюда следует, что количество различных слов $\beta$, которые могут составить множество $W$ не превосходит $((r d+r) d)^{(r d+r) d}$, а число таких множеств не превосходит $2^{((r d+r) d)^{(r d+r) d}}$

Лемма доказана.

Пусть $P \in D^{\prime}(n, m, T,(\alpha))$. Заметим, что в каждом вершинном пути $\pi^{\prime}\left(q_{r}, \alpha\right)$, определяемом по графу $P$, вершины можно пронумеровать номерами от 1 до $[T]+1$ в порядке появления этих вершин в пути так, что вершина $q_{r}$ получит первый номер. Разобьем каждый класс $D^{\prime}\left(n, m, T,\left(\alpha_{i_{1}}, \alpha_{i_{2}}, \ldots, \alpha_{i_{k}}\right)\right)$ на подклассы $D^{\prime}\left(n, m, T,\left(\alpha_{i_{1}}, \alpha_{i_{2}}, \ldots, \alpha_{i_{k}}\right), \Xi\right)$ по типу $\Xi$ взаимного расположения путей $\pi\left(q_{r}, \alpha_{i_{j}}\right)$, где $r \in\{1,2\}, j \in\{1,2, \ldots, k\}$. Тип $\Xi$ определяется следуюшим образом. Пусть $P \in D^{\prime}\left(n, m, T,\left(\alpha_{i_{1}}, \alpha_{i_{2}}, \ldots, \alpha_{i_{k}}\right)\right)$. Без ограничения общности полагаем $i_{1}<i_{2}<\ldots<i_{k}$. Пусть теперь

$$
\Xi(P)=\left(F_{1}, F_{2}, \ldots, F_{k-1}\right)
$$

- упорядоченный набор наборов, где каждый из наборов $F_{h}$ есть упорядоченный набор $\left(L_{h}^{1}, L_{h}^{2}, \ldots, L_{h}^{r}\right)$ упорядоченных наборов четверок $\left(s_{1}, s_{2}, s_{3}, s_{4}\right)$, определяемая следующим образом. Положим $S=\tilde{\pi}\left(q_{1}, \alpha_{i_{h+1}}\right)$. Далее пусть $S^{\prime}$ - подмножество тех вершин из $S$, в которые ведет на графе $P$ по крайней мере одно ребро, встречающееся в путях $\pi\left(q_{s}, \alpha_{i_{j}}\right)$ при $s \in\{1,2, \ldots, r\}$ и $j \leqslant h$, которое отлично от ребра, которое ведет в эту вершину на пути $\pi\left(q_{1}, \alpha_{i_{h+1}}\right)$. Для каждого $q \in S$ определим четверку $\left(s_{1}, s_{2}, s_{3}, s_{4}\right)$ следующим образом: $s_{1}$ - номер вершины $q$ в вершинном пути $\pi^{\prime}\left(q_{1}, \alpha_{i_{h+1}}\right)$, в том смысле о котором говорилось ранее. По определению $S^{\prime}$ существует такое $p \leqslant h$, что $q \in \pi^{\prime}\left(q_{s}, \alpha_{i_{p}}\right)$. Пусть теперь $s_{2}$ - номер вершины $q$ в $\pi^{\prime}\left(q_{r}, \alpha_{i_{p}}\right), s_{3}=s, s_{4}=p$. Выписывая теперь полученные четверки в порядке возрастания $s_{1}$, получаем набор $L_{h}^{1}$. В случае, если $S^{\prime}=\varnothing$, полагаем $L_{h}^{1}$ равным пустому набору. Набор $L_{h}^{z}$ при $2 \leqslant z$ определяется аналогично, только полагаем теперь $S=\tilde{\pi}\left(q_{z}, \alpha_{i_{h+1}}\right)$ и при определении $S^{\prime}$ рассматриваем теперь пути $\pi\left(q_{s}, \alpha_{i_{j}}\right)$ при $s \in\{1,2, \ldots, r\}$ и $j \leqslant h$ и $\pi\left(q_{z}^{\prime}, \alpha_{i_{h+1}}\right)$ при $z^{\prime}<z$. Нетрудно видеть, что подклассы $D^{\prime}\left(n, m, T,\left(\alpha_{i_{1}}, \alpha_{i_{2}}, \ldots, \alpha_{i_{k}}\right), \Xi_{1}\right), D^{\prime}\left(n, m, T,\left(\alpha_{i_{1}}, \alpha_{i_{2}}, \ldots, \alpha_{i_{k}}\right), \Xi_{2}\right)$ не пересекаются при разных $\Xi_{1}$ и $\Xi_{2}$, то есть мы имеем дело с разбиением класса $D^{\prime}\left(n, m, T,\left(\alpha_{i_{1}}, \alpha_{i_{2}}, \ldots, \alpha_{i_{k}}\right)\right)$ на непересекающиеся подклассы.

Рассмотрим граф $P \in D^{\prime}\left(n, m, T,\left(\alpha_{i_{1}}, \alpha_{i_{2}}, \ldots, \alpha_{i_{k}}\right), \Xi\right)$. Заметим , что общее число четверок в наборах $L_{i}^{s}$ при $1 \leqslant i \leqslant k-1$ и $1 \leqslant s \leqslant r$, образующих тип $\Xi$, если их выписать подряд, равно $\mathrm{gl}\left(P,\left(\alpha_{i_{1}}, \alpha_{i_{2}}, \ldots, \alpha_{i_{k}}\right)\right)-(r-1)$. Стало быть, типу $\Xi$ можно приписать значение $\operatorname{gl}\left(\Xi,\left(\alpha_{i_{1}}, \alpha_{i_{2}}, \ldots, \alpha_{i_{k}}\right)\right)$. Зафиксируем множество слов $\left(\alpha_{i_{1}}, \alpha_{i_{2}}, \ldots, \alpha_{i_{k}}\right)$ и число $p$. Нетрудно оценить сверху число подклассов $D^{\prime}\left(n, m, T,\left(\alpha_{i_{1}}, \alpha_{i_{2}}, \ldots, \alpha_{i_{k}}\right), \Xi\right)$, для котоpых $\operatorname{gl}\left(\Xi,\left(\alpha_{i_{1}}, \alpha_{i_{2}}, \ldots, \alpha_{i_{k}}\right)\right)=p$. Это количество не превосходит $p^{r k}\left(r d(T+1)^{2}\right)^{p}$, что вытекает из сделанного замечания относительно общего числа четверок в наборах $L_{i}^{s}$, а также оценки числа четверок того вида, который был обозначен при описании понятия типа. Зафиксируем $k$ и множество $\left(\alpha_{i_{1}}, \alpha_{i_{2}}, \ldots, \alpha_{i_{k}}\right)$ из $k$ слов длины [T]. При этом полагаем, что $i_{1}<i_{2}<\ldots<i_{k}$. Зафиксируем также тип $\Xi$ взаимного расположения путей $\alpha_{i_{j}}$. Введем обозначение

$$
p_{\alpha_{i_{1}}, \alpha_{i_{2}}, \ldots, \alpha_{i_{k}}, \Xi}=\frac{\left|D^{\prime}\left(n, m, T,\left(\alpha_{i_{1}}, \alpha_{i_{2}}, \ldots, \alpha_{i_{k}}\right), \Xi\right)\right|}{|D(n, m)|} .
$$

Докажем следующее утверждение. 
Лемма 5. Пусть $\operatorname{gl}\left(\Xi,\left(\alpha_{i_{1}}, \alpha_{i_{2}}, \ldots, \alpha_{i_{k}}\right)=s\right.$. Тогдa

$$
p_{\alpha_{i_{1}}, \alpha_{i_{2}}, \ldots, \alpha_{i_{k}}, \Xi} \leqslant \frac{1}{n^{s}} \text {. }
$$

Доказательство. Пусть имеется некоторая стохастическая процедура $\mathfrak{I}$ построения графов из $D(n, m)$, которая с одинаковой вероятностью приводит к построению любого графа

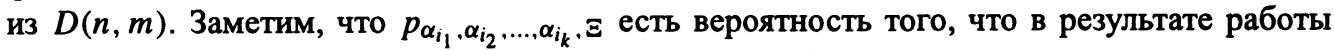
этой стохастической процедуры получится граф из $D^{\prime}\left(n, m, T,\left(\alpha_{i_{1}}, \alpha_{i_{2}}, \ldots, \alpha_{i_{k}}\right), \Xi\right)$. Рассмотрим следующий процесс $\mathfrak{J}_{0}$ построения графа из $D(n, m)$. Процесс $\mathfrak{I}_{0}$ состоит из двух этапов. Первый этап состоит из $r$ [T] $k$ последовательных шагов и определяется следующим образом. Перед началом процесса имеются множество $Q$, вершины $q_{i} \in Q$ при $1 \leqslant i \leqslant r$, текущее множество ребер $S=\varnothing$, текущая вершина $q^{1}=q_{1}$. Пусть $a-$ первый символ слова $\alpha_{i_{1}}$. Тогда первый шаг процесса состоит в равновероятном выборе любой из вершин множества $Q$ в качестве концевой вершины ребра, исходящего из текущей вершины $q=q_{1}$ и помеченного символом $a$. Обозначим выбранную вершину $q^{1 \prime}$ и сделаем ее текущей для второго шага, то есть положим $q^{2}=q^{11}$, а текущее множество ребер пополним построенным помеченным ребром. Пусть проделаны $i<[T]$ шагов процесса. На $(i+1)$-м шаге рассматриваем текущую вершину $q^{i}$ и $(i+1)$-ю букву слова $\alpha_{i_{1}}$. Пусть это буква $a^{\prime}$. Тогда, если среди ребер текущего множества $S$ нет ребра, исходящего из вершины $q^{i}$ и помеченного символом $a^{\prime}$, то на $(i+1)$-м шаге осуществляется равновероятный выбор вершины из $Q$, которую мы обозначим $q^{i \prime}$ и которая становится текущей для следуюшего шага, то есть $q^{i+1}=q^{i}$, а также добавление в множество $S$ ребра, исходящего из вершины $q^{i}$, помеченного символом $a^{\prime}$ и входящего в $q^{i \prime}$, в противном случае множество $S$ остается прежним, а текущей вершиной становится та, в которую ведет ребро из $S$, помеченное символом $a$, первая вершина которого есть $q^{i}$. По окончании первых $[T]$ шагов текущей вершиной назначается вершина $q_{2}$, то есть $q^{[T]}+1=q_{2}$, и процесс продолжается по тем же правилам. По окончании следующих $[T]$ шагов текущей вершиной назначается $q_{3}$ и так далее. После первых $r[T]$ шагов текущей вершиной снова назначается $q_{1}$ и процесс продолжается с заменой слова $\alpha_{i_{1}}$ на $\alpha_{i_{2}}$. Еще через $r[T]$ шагов слово $\alpha_{i_{2}}$ заменяется на слово $\alpha_{i_{3}}$ и т. д.

Второй этап состоит в последовательном просмотре вершин множества $Q$ и выполнения для каждой из них следующей операции: если для некоторого символа $a$ в множестве $S$ нет ребра, ведущего из вершины $q$ и помеченного символом $a$, то производится случайный выбор вершины $q^{\prime}$ и добавление в множество $S$ ребра, помеченного символом $a$, начинающегося в $q$ и ведущего в $q^{\prime}$. Второй этап заканчивается, когда множество ребер $S$ вместе с множеством $Q$ образуют некоторый граф из $D(n, m)$, который и выдается на выходе стохастической процедуры $\Im_{0}$.

Нетрудно видеть, что описанный процесс с равной вероятностью приводит к построению любого графа из $D(n, m)$.

Посмотрим теперь, какие ограничения на работу процесса $\Im_{0}$ следует наложить, чтобы на его выходе появился граф из $D^{\prime}\left(n, m, T,\left(\alpha_{i_{1}}, \alpha_{i_{2}}, \ldots \alpha_{i_{k}}\right)\right.$, $\left.\Xi\right)$. Для $i<2[T]$ необходимо потребовать, чтобы $q^{i \prime} \neq q^{j \prime}$ для $j<i$ и $q^{i \prime} \neq q_{s}$ при $1 \leqslant s \leqslant r$. На шаге с номером 2[T] необходимо потребовать, чтобы $q^{2[T] \prime}=q^{[T]^{\prime}}$. При $l[T]<i<(l+1)[T], 2 \leqslant l \leqslant r-1$, необходимо требовать, чтобы $q^{i \prime} \neq q^{j \prime}$ для $j<i$ и $q^{i \prime} \neq q_{s}$ при $1 \leqslant s \leqslant r$, наконец, при $i=l[T], 3 \leqslant l \leqslant r$, необходимо, чтобы $q^{l[T]^{\prime}}=q^{[T]^{\prime}}$.

Пусть теперь

$$
\Xi=\left(\left(L_{1}^{1}, L_{1}^{2}, \ldots L_{1}^{r}\right),\left(L_{2}^{1}, L_{2}^{2}, \ldots L_{2}^{r}\right), \ldots,\left(L_{k-1}^{1}, L_{k-1}^{2}, \ldots, L_{k-1}^{r}\right)\right.
$$


и $\left(s_{1}, s_{2}, s_{3}, s_{4}\right) \in L_{h}^{s}$ для некоторых $1 \leqslant h \leqslant k-1$ и $1 \leqslant s \leqslant r$. Тогда четверка $\left(s_{1}, s_{2}, s_{3}, s_{4}\right)$ накладывает ограничение на процедуру $\mathfrak{\Im}_{0}$, состоящее в том, что на шаге с номером $i=r[T] h+(s-1)[T]+s 1-1$ выбираемая вершина $q^{i \prime}$ однозначно определяется тройкой $\left(s_{2}, s_{3}, s_{4}\right)$, а именно, $q^{i \prime}=q^{j}$, где $j=r[T]\left(s_{4}-1\right)+[T]\left(s_{3}-1\right)+s_{2}$. Это вытекает непосредственно из определения типа $\Xi$. Так как общее число четверок в наборах $L_{i}^{r}$ ровно $p-(r-1)$, указанные ограничения вместе с аналогичным ограничениями на шагах с номерами $l[T]$ при $2 \leqslant l \leqslant r$ образуют систему из $p$ ограничений, каждое из которых состоит в том, что на некотором шаге $I$ процесса $\Im_{0}$ ребро, определяемое на этом шаге не содержится среди ребер текущего множества ребер I-го шага и направляется в некоторую однозначно определенную вершину. Нетрудно понять, что каждое такое ограничение приводит к уменышению вероятности появления графа $P \in D^{\prime}\left(n, m, T,\left(\alpha_{i_{1}}, \alpha_{i_{2}}, \ldots, \alpha_{i_{k}}\right), \Xi\right)$ в $n$ раз, отсюда следует, что

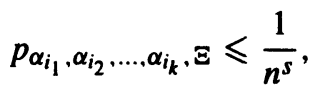

что и требовалось доказать.

\section{6. Доказательство теоремы 4}

Рассмотрим граф $P$ из $D(n, m)$ и $r$ вершин $q_{i_{1}}, q_{i_{2}}, \ldots, q_{i_{r}}$ из $Q$. Если найдется хотя бы одно слово $\alpha$ в алфавита $A$ такое, что

$$
f_{P}\left(q_{i_{1}}, \alpha\right)=f_{P}\left(q_{i_{2}}, \alpha\right)=\ldots=f_{P}\left(q_{i_{r}}, \alpha\right)
$$

то длину кратчайшего такого слова назовем временем склейки вершин $q_{i_{1}}, q_{i_{2}}, \ldots, q_{i_{r}}$ в графе $P$ и обозначим $T\left(P, q_{i_{1}}, q_{i_{2}}, \ldots, q_{i_{r}}\right)$. Если же такого слова $\alpha$ не существует, то положим $T\left(P, q_{i_{1}}, q_{i_{2}}, \ldots, q_{i_{r}}\right)=0$.

Рассмотрим многообразие наборов вида $\left(P,\left(q_{i_{1}}, q_{i_{2}}, \ldots, q_{i_{r}}\right)\right)$, где $P \in D(n, m)$, а $\left(q_{i_{1}}, q_{i_{2}}, \ldots, q_{i_{r}}\right)$ - неупорядоченный набор $r$ различных элементов множества $Q$. Это многообразие наборов мы обозначим $\mathfrak{R}(n, m)$. Выделим в этом многообразии подмногообразие $\mathfrak{R}\left(n, m, T_{1}, T_{2}\right)$ таких наборов $\left(P,\left(q_{i_{1}}, q_{i_{2}}, \ldots, q_{i_{r}}\right)\right)$, что время склейки состояний $q_{i_{1}}, q_{i_{2}}, \ldots, q_{i_{r}}$ в графе $P$ заключено в пределах от $T_{2}$ до $T_{1}$. Рассмотрим функцию

$$
p\left(n, m, T_{1}, T_{2}\right)=\frac{\left|\mathfrak{R}\left(n, m, T_{1}, T_{2}\right)\right|}{|\mathfrak{R}(n, m)|} .
$$

Положим

$$
T_{2}(n)=\log _{m} n-4 \log _{2} \log _{2} n, \quad T_{1}(n)=\log _{m} n+\log _{2} \log _{2} \log _{2} \log _{2} \log _{2} n .
$$

Мы докажем теорему 4, если покажем, что $p\left(n, m, T_{1}(n), T_{2}(n)\right) \rightarrow 1$ при $n \rightarrow \infty$.

Заметим теперь, что многообразия $\mathfrak{R}(n, m)$ и $\mathfrak{R}\left(n, m, T_{1}, T_{2}\right)$ разбиваются на $\left(\begin{array}{l}n \\ r\end{array}\right)$ равномощных классов наборов, соответствующих фиксации $r$ состояний. Обозначим классы, соответствующие набору $\left(q_{1}, q_{2}, \ldots, q_{r}\right)$ соответственно $\mathfrak{R}^{\prime}(n, m)$ и $\mathfrak{R}^{\prime}\left(n, m, T_{1}, T_{2}\right)$. Положим теперь

$$
p^{\prime}\left(n, m, T_{1}, T_{2}\right)=\frac{\left|\mathfrak{R}^{\prime}\left(n, m, T_{1}, T_{2}\right)\right|}{\left|\mathfrak{R}^{\prime}(n, m)\right|} .
$$

Очевидно, что $p^{\prime}\left(n, m, T_{1}, T_{2}\right)=p\left(n, m, T_{1}, T_{2}\right)$, так что для доказательства теоремы 4 достаточно показать, что $p^{\prime}\left(n, m, T_{1}(n), T_{2}(n)\right) \rightarrow 1$ при $n \rightarrow \infty$. 
Обозначим $D\left(n, m, T^{\prime}\right)$ подмножество $D(n, m)$, состоящее из таких графов $P$, что $T\left(P, q_{1}, q_{2}, \ldots, q_{r}\right) \leqslant T^{\prime}$. Положим

$$
p_{1}^{\prime}(n, m, T)=\frac{|D(n, m, T)|}{|D(n, m)|} .
$$

Покажем, что при $n \rightarrow \infty$

$$
p_{1}^{\prime}\left(n, m, T_{1}(n)\right) \rightarrow 1, \quad p_{1}^{\prime}\left(n, m, T_{2}(n)\right) \rightarrow 0
$$

Очевидно, что доказав эти утверждения, мы тем самым докажем теорему 4.

Докажем первое утверждение. Положим $K_{1}=m^{\left[T_{1}\right]}$. Тогда

$$
K_{1}=n^{r-1} L_{1}(n)
$$

где

$$
m\left(\log _{2} \log _{2} \log _{2} \log _{2} n\right)^{\log _{2} m} \geqslant L_{1}(n) \geqslant \frac{\log _{2} \log _{2} \log _{2} \log _{2} n}{m}
$$

Занумеруем все слова в алфавите $A$ длины $\left[T_{1}\right]$ числами от 1 до $K_{1}: \alpha_{1}, \alpha_{2}, \ldots, \alpha_{K_{1}}$. Обозначим $N_{\alpha_{i_{1}}, \alpha_{i_{2}} \ldots ., \alpha_{i_{k}}}$ мощность множества $D^{\prime}\left(n, m, T_{1},\left(\alpha_{i_{1}}, \alpha_{i_{2}}, \ldots, \alpha_{i_{k}}\right)\right)$. Согласно формуле включений и исключений

$$
\begin{aligned}
\left|D^{\prime}\left(n, m, T_{1}\right)\right|= & \sum_{i_{1}=1}^{K_{1}} N_{\alpha_{i_{1}}}-\sum_{1 \leqslant i_{1}<i_{2} \leqslant K_{1}} N_{\alpha_{i_{1}}, \alpha_{i_{2}}}+\ldots \\
& +(-1)^{k} \sum_{1 \leqslant i_{1}<i_{2}<\ldots<i_{k} \leqslant K_{1}} N_{\alpha_{i_{1}}, \alpha_{i_{2}} \ldots . \alpha_{i_{k}}}+\ldots+(-1)^{K_{1}} N_{\alpha_{1}, \alpha_{2}, \ldots, \alpha_{K_{1}}} .
\end{aligned}
$$

Пусть $d$ - максимальное целое четное число, не превосходящее $\log _{2} \log _{2} \log _{2} n$. Тогда согласно неравенству Бонферрони [3]

$$
\begin{aligned}
&\left|D^{\prime}\left(n, m, T_{1}\right)\right| \geqslant \sum_{i_{1}=1}^{K_{1}} N_{\alpha_{i_{1}}}-\sum_{1 \leqslant i_{1}<i_{2} \leqslant K_{1}} N_{\alpha_{i_{1}}, \alpha_{i_{2}}}+\ldots \\
&+(-1)^{d} \sum_{1 \leqslant i_{1}<i_{2}<\ldots<i_{d} \leqslant K_{1}} N_{\alpha_{i_{1}}, \alpha_{i_{2}}, \ldots, \alpha_{i_{d}}} .
\end{aligned}
$$

Теперь для каждого набора слов $\left(\alpha_{i_{1}}, \alpha_{i_{2}}, \ldots, \alpha_{i_{k}}\right)$ справедливо равенство

$$
N_{\alpha_{i_{1}}, \alpha_{i_{2}}, \ldots . \alpha_{i_{k}}}=N_{\alpha_{i_{1}}, \alpha_{i_{2}}, \ldots . \alpha_{i_{k}}}^{1}+N_{\alpha_{i_{1}}, \alpha_{i_{2}} \ldots ., \alpha_{i_{k}}}^{2}+N_{\alpha_{i_{1}}, \alpha_{i_{2}}, \ldots . \alpha_{i_{k}}}^{3},
$$

где каждое из трех слагаемых обозначает число графов в соответствующем подклассе $D_{i}^{\prime}\left(n, m, T_{1},\left(\alpha_{i_{1}}, \alpha_{i_{2}}, \ldots, \alpha_{i_{k}}\right)\right), 1 \leqslant i \leqslant 3$. При нечетных $k$

$$
N_{\alpha_{i_{1}}, \alpha_{i_{2}} \ldots . \alpha_{i_{k}}} \geqslant N_{\alpha_{i_{1}}, \alpha_{i_{2}}, \ldots . \alpha_{i_{k}}}^{1}
$$


Так что из неравенства (2) следует неравенство

$$
\begin{aligned}
\left|D^{\prime}\left(n, m, T_{1}\right)\right| \geqslant \sum_{i_{1}=1}^{K_{1}} N_{\alpha_{i_{1}}}^{1}-\sum_{1 \leqslant i_{1}<i_{2} \leqslant K_{1}} N_{\alpha_{i_{1}}, \alpha_{i_{2}}}^{1}+\ldots \\
+(-1)^{d+1} \sum_{1 \leqslant i_{1}<i_{2}<\ldots<i_{d} \leqslant K_{1}} N_{\alpha_{i_{1}}, \alpha_{i_{2}}, \ldots ., \alpha_{i_{d}}}^{1} \sum_{2 \leqslant p \leqslant d} \sum_{1 \leqslant i_{1}<i_{2}<\ldots<i_{p} \leqslant K_{1}} N_{\alpha_{i_{1}}, \alpha_{i_{2}}, \ldots . \alpha_{i_{p}}}^{2} \sum_{2 \leqslant p \leqslant d} \sum_{1 \leqslant i_{1}<i_{2}<\cdots<i_{p} \leqslant K_{1}} N_{\alpha_{i_{1}}, \alpha_{i_{2}} \ldots \ldots, \alpha_{i_{p}}}^{3}
\end{aligned}
$$

где суммирование в соответствующих суммах проводится по четным значениям $p$.

Введем обозначения

$$
\begin{aligned}
p\left(n, m, T_{1}\right) & =\frac{\left|D\left(n, m, T_{1}\right)\right|}{|D(n, m)|} \\
p\left(n, m, T_{1}, \alpha_{i_{1}}, \alpha_{i_{2}}, \ldots, \alpha_{i_{k}}\right) & =\frac{N_{\alpha_{i_{1}}, \alpha_{i_{2}}, \ldots, \alpha_{i_{k}}}}{|D(n, m)|} \\
p^{1}\left(n, m, T_{1}, \alpha_{i_{1}}, \alpha_{i_{2}}, \ldots, \alpha_{i_{k}}\right) & =\frac{N_{\alpha_{i_{1}}, \alpha_{i_{2}}, \ldots, \alpha_{i_{k}}}^{1}}{|D(n, m)|} \\
p^{2}\left(n, m, T_{1}, \alpha_{i_{1}}, \alpha_{i_{2}}, \ldots, \alpha_{i_{k}}\right) & =\frac{N_{\alpha_{i_{1}}, \alpha_{i_{2}}, \ldots . \alpha_{i_{k}}}^{2}}{|D(n, m)|} \\
p^{3}\left(n, m, T_{1}, \alpha_{i_{1}}, \alpha_{i_{2}}, \ldots, \alpha_{i_{k}}\right) & =\frac{N_{\alpha_{i_{1}}, \alpha_{i_{2}}, \ldots . \alpha_{i_{k}}}^{3}}{|D(n, m)|}
\end{aligned}
$$

Из неравенства (3) следует, что

$$
\begin{aligned}
\left|p\left(n, m, T_{1}\right)\right| \geqslant & \sum_{i_{1}=1}^{K_{1}} p_{\alpha_{i_{1}}}^{1}-\sum_{1 \leqslant i_{1}<i_{2} \leqslant K_{1}} p_{\alpha_{i_{1}}, \alpha_{i_{2}}}^{1}+\ldots \\
& +(-1)^{d+1} \sum_{1 \leqslant i_{1}<i_{2}<\ldots<i_{d} \leqslant K_{1}} p_{\alpha_{i_{1}}, \alpha_{i_{2}} \ldots . . \alpha_{i_{d}}}^{1} \sum_{2 \leqslant p \leqslant d 1 \leqslant i_{1}<i_{2}<\ldots<i_{p} \leqslant K_{1}} p_{\alpha_{i_{1}}, \alpha_{i_{2}} \ldots . . \alpha_{i_{p}}}^{2} \sum^{2 \leqslant p \leqslant d 1 \leqslant i_{1}<i_{2}<\ldots<i_{p} \leqslant K_{1}}
\end{aligned}
$$

где суммирование в соответствуюших суммах проводится по четным значениям $p$, $2 \leqslant p \leqslant d$.

Докажем, что

$$
\sum_{2 \leqslant p \leqslant d} \sum_{1 \leqslant i_{1}<i_{2}<\ldots<i_{p} \leqslant K_{1}} p_{\alpha_{i_{1}}, \alpha_{i_{2}} \ldots . . \alpha_{i_{p}}}^{2}+\sum_{2 \leqslant p \leqslant d \leqslant i_{1}<i_{2}<\ldots<i_{p} \leqslant K_{1}} \sum_{\alpha_{i_{1}}, \alpha_{i_{2}}, \ldots . \alpha_{i_{p}}}^{3}=o(1),
$$


где суммирование в соответствующих суммах, как и выше, проводится по четным значениям $p, 2 \leqslant p \leqslant d$ и

$$
\sum_{i_{1}=1}^{K_{1}} p_{\alpha_{i_{1}}}^{1}-\sum_{1 \leqslant i_{1}<i_{2} \leqslant K_{1}} p_{\alpha_{i_{1}}, \alpha_{i_{2}}}^{1}+\ldots+(-1)^{d+1} \sum_{1 \leqslant i_{1}<i_{2}<\ldots<i_{d} \leqslant K_{1}} p_{\alpha_{i_{1}}, \alpha_{i_{2}}, \ldots, \alpha_{i_{d}}}^{1}=1+o(1) .
$$

Отсюда и из (4) следует первое утверждение теоремы.

Рассмотрим теперь суммы

$$
\begin{aligned}
& S_{1}=\sum_{2 \leqslant p \leqslant d \leqslant i_{1}<i_{2}<\ldots<i_{p} \leqslant K_{1}} \sum_{\alpha_{i_{1}}, \alpha_{i_{2}} \ldots . . \alpha_{i_{p}}}^{2}, \\
& S_{2}=\sum_{2 \leqslant p \leqslant d} \sum_{1 \leqslant i_{1}<i_{2}<\ldots<i_{p} \leqslant K_{1}} N_{\alpha_{i_{1}}, \alpha_{i_{2}}, \ldots, \alpha_{i_{p}}}^{3},
\end{aligned}
$$

где здесь и ниже суммирование в соответствующих суммах проводится по четным $p$, $2 \leqslant p \leqslant d$.

Положим

$$
D=2^{((r d+r) d)^{(r d+r) d}}
$$

и продублируем $D$ раз каждую внутреннюю сумму в $S_{1}$, то есть перейдем от $S_{1}$ к $S_{3}=D S_{2}$. Из лемм 3 и 4 следует, что если граф $P$ подсчитывался $s$ раз в $S_{2}$, то в $S_{3}$ он будет посчитан не менее $s$ раз, откуда $S_{2} \leqslant S_{3}$. Итак,

$$
\begin{aligned}
\sum_{2 \leqslant p \leqslant d} \sum_{1 \leqslant i_{1}<i_{2}<\ldots<i_{p} \leqslant K_{1}} N_{\alpha_{i_{1}}, \alpha_{i_{2}} \ldots \ldots \alpha_{i_{p}}}^{2} & +\sum_{2 \leqslant p \leqslant d 1 \leqslant i_{1}<i_{2}<\ldots<i_{p} \leqslant K_{1}} \sum_{\alpha_{i_{1}}, \alpha_{i_{2}}, \ldots, \alpha_{i_{p}}}^{3} \sum_{2 \leqslant p \leqslant d \leqslant i_{1}<i_{2}<\ldots<i_{p} \leqslant K_{1}} N_{\alpha_{i_{1}}, \alpha_{i_{2}}, \ldots, \alpha_{i_{p}}}^{2} .
\end{aligned}
$$

Отсюда следует, что для проверки асимптотики (5) достаточно доказать, что

$$
(D+1) \sum_{2 \leqslant s \leqslant d \leqslant i_{1}<i_{2}<\ldots<i_{s} \leqslant K_{1}} \sum_{\alpha_{i_{1}}, \alpha_{i_{2}}, \ldots, \alpha_{i s}}^{2}=o(1) .
$$

Перепишем теперь сумму

$$
\sum_{2 \leqslant s \leqslant d \leqslant i_{1}<i_{2}<\ldots<i_{s} \leqslant K_{1}} \sum_{\alpha_{i_{1}}, \alpha_{i_{2}} \ldots ., \alpha_{i s}}^{2}
$$

в виде

$$
\sum_{2 \leqslant s \leqslant d} \sum_{1 \leqslant i_{1}<i_{2}<\ldots<i_{s} \leqslant K_{1}} \sum_{s^{\prime}>s} \sum_{\left(\Xi: \operatorname{gl}\left(\Xi,\left(\alpha_{i_{1}}, \alpha_{i_{2}}, \ldots, \alpha_{i_{s}}\right)\right)=s^{\prime}\right)} p_{\alpha_{i_{1}}, \alpha_{i_{2}}, \ldots, \alpha_{i_{s}}, \Xi .}
$$

Теперь, воспользовавшись леммой 5 , а также указанной оценкой числа типов $\Xi \mathrm{c}$

$$
\operatorname{gl}\left(\Xi,\left(\alpha_{i_{1}}, \alpha_{i_{2}}, \ldots, \alpha_{i_{s}}\right)\right)=s^{\prime},
$$

можно оценить последнюю сумму сверху суммой

$$
\sum_{2 \leqslant s \leqslant d} K_{1}^{s} \sum_{s^{\prime}>s} \frac{\left.\left(s^{\prime}\right)^{r s}\left(r d\left(T_{1}+1\right)^{2}\right)^{s^{\prime}}\right)}{n^{s^{\prime}}}
$$


Учитывая, что

$$
\begin{aligned}
d & \leqslant \log _{2} \log _{2} \log _{2} n, \\
T_{1} & \leqslant \log _{m} n+\log _{2} \log _{2} \log _{2} \log _{2} \log _{2} n, \\
K_{1} & \leqslant n\left(\log _{2} \log _{2} \log _{2} \log _{2} n\right)^{m},
\end{aligned}
$$

и используя обычную технику асимптотических оценок, нетрудно убедиться в том, что последняя сумма есть $O(1 / \sqrt{n})$. Так как

$$
(D+1) O\left(\frac{1}{\sqrt{n}}\right)=o(1),
$$

асимптотика (7) доказана.

Наконец, перейдем к асимптотике (6).

Нетрудно получить, что при всех $k \leqslant d$

$$
\frac{1}{n^{k(r-1)}}\left(1-\frac{\log _{2}^{2} n}{n}\right) \leqslant p_{\alpha_{i_{1}}, \alpha_{i_{2}}, \ldots, \alpha_{i_{k}}}^{1} \leqslant \frac{1}{n^{k(r-1)}},
$$

так что

$$
\begin{aligned}
\sum_{i_{1}=1}^{K_{1}} p_{\alpha_{i_{1}}}^{1}-\sum_{1 \leqslant i_{1}<i_{2} \leqslant K_{1}} p_{\alpha_{i_{1}}, \alpha_{i_{2}}}^{1}+\ldots+(-1)^{d+1} & \sum_{1 \leqslant i_{1}<i_{2}<\ldots<i_{d} \leqslant K_{1}} p_{\alpha_{i_{1}}, \alpha_{i_{2}}, \ldots, \alpha_{i_{d}}}^{1} \sum_{1 \leqslant k \leqslant d} \frac{\left(\begin{array}{c}
K_{1} \\
k
\end{array}\right)(-1)^{k}\left(1-\delta(k)\left(\log _{2}^{2} n\right) / n\right)}{k ! n^{k(r-1)}}, \\
& \geqslant \sum_{1 \leqslant k},
\end{aligned}
$$

где $\delta(k)=1$ при нечетных $k$ и $\delta(k)=0$ при четных $k$. Далее, при четных $k$

$$
\left(\begin{array}{c}
K_{1} \\
k
\end{array}\right) \leqslant \frac{K_{1}^{k}}{k !}
$$

При нечетных $k \leqslant d$ нетрудно вывести, что

$$
\left(\begin{array}{c}
K_{1} \\
k
\end{array}\right) \geqslant \frac{K_{1}^{k}\left(1-\left(\log _{2} n\right) / n\right)}{k !},
$$

Tak что

$$
\begin{aligned}
\sum_{i_{1}=1}^{K_{1}} p_{\alpha_{i_{1}}}^{1}-\sum_{1 \leqslant i_{1}<i_{2} \leqslant K_{1}} p_{\alpha_{i_{1}}, \alpha_{i_{2}}}^{1}+\ldots & +(-1)^{d+1} \sum_{1 \leqslant i_{1}<i_{2}<\ldots<i_{d} \leqslant K_{1}} p_{\alpha_{i_{1}}, \alpha_{i_{2}}, \ldots, \alpha_{i_{d}}}^{1} \sum \sum_{1 \leqslant k \leqslant d}(-1)^{k} \frac{\left(K_{1} / n^{r-1}\right)^{k}}{k !}+o(1) \\
& =1-\exp \left(-\left(K_{1} / n^{r-1}\right)\right)+o(1)=1+o(1) .
\end{aligned}
$$

Итак, асимптотика (6), а вместе с ней и первое утверждение теоремы 4, полностью доказаны. 
Перейдем к второму утверждению теоремы. Обозначим $D(n, m, \alpha)$ множество графов из $P$, для которых вершинные пути $\pi^{\prime}\left(q_{1}, \alpha\right)$ и $\pi\left(q_{2}, \alpha\right)$ имеют общую последнюю вершину. Обозначим $l(\alpha)$ длину слова $\alpha$. Тогда

$$
\left|D\left(n, m, T_{2}\right)\right| \leqslant \sum_{\alpha, l(\alpha) \leqslant T_{2}}|D(n, m, \alpha)| .
$$

С помощью рассуждений, аналогичных тем, которые использовались в лемме 4, нетрудно установить, что для всякого $\alpha$ такого, что $l(\alpha) \leqslant T_{2}$,

$$
\frac{|D(n, m, \alpha)|}{|D(n, m)|} \leqslant \frac{\log _{2}^{3} n}{n} \text {. }
$$

Учтем теперь, что различных слов длины, не превосходящей $T_{2}$, не больше, чем $\mathrm{mm}^{T_{2}}$. Отсюда легко выводим, что

$$
\frac{\left|D\left(n, m, T_{2}\right)\right|}{|D(n, m)|}=o(1)
$$

Этим доказывается второе утверждение теоремы 4.

В заключение автор приносит искреннюю благодарность В. Б. Кудрявцеву и А. С. Подколзину за поддержку и помощь в работе.

\section{Список литературы}

1. Кудрявцев В. Б., Алешин С. В., Подколзин А. С., Введение в теорию автоматов. Наука, Москва, 1985.

2. Соколовский М. Н., Сложность порождения подстановок и эксперименты с автоматами. Дискретный анализ (1976) 29, 68-86.

3. Феллер В., Введение в теорию вероятностей и ее приложения. Мир, Москва, 1984.

4. Hibbard T. N., Least upper bounds on minimal terminal state experiments for two classes of sequential machines. J. Assoc. Comput. Mach. (1961) 8, 601-612.

Статья поступила 10.10.2002. 